\title{
Nanoquímica, un campo de conocimientos de alto valor educativo y disciplinar
}

\section{Nanochemistry, a field of knowledge with high educational and disciplinary value}

\author{
Jorge Meinguer Ledesma*
}

\begin{abstract}
Nanochemistry is a field of knowledge focused on obtaining and characterizing the materials at the nanoscale. It is based on bottom-up synthesis methods that involve molecular self-assembly processes, as well as tools, models and approaches that are specific to chemistry. In the last decades, this subdiscipline has made valuable contributions to the fields of research, to the industry, technology and to the environmental care. In this work, the historical development, basic notions, as well as some representative techniques and applications of nanochemistry are analyzed in order to show its disciplinary value. In general, the educational proposals referenced in this documentary research allow to accentuate the multi and interdisciplinary nature of nanochemistry. Subsequently, aspects that allow justifying its educational relevance are discussed. The last part of this work offers a description of various proposals that have been developed in high school and in the initial stages of university education regarding the teaching of contents related to nanochemistry, since these can guide and strengthen the educational work in this field.
\end{abstract}

KEYWORDS: nanochemistry, chemistry teaching, chemistry curriculum, nanotechnology education, bottom-up approach.

RESUMEN: La nanoquímica es un área de conocimientos enfocada en la obtención y la caracterización de materiales en la escala nanométrica. Se fundamenta en métodos de síntesis ascendente (bottom-up) que involucran procesos de autoensamblaje molecular, así como herramientas, modelos y aproximaciones que son propios de la química. En las últimas décadas esta subdisciplina ha hecho valiosas contribuciones a los ámbitos de la investigación, la industria, la tecnología y el cuidado del medio ambiente. En este trabajo, se analiza el desarrollo histórico, las nociones básicas, asi como algunas técnicas y aplicaciones representativas de la nanoquímica con la finalidad de mostrar su valor disciplinar. En general, las propuestas educativas referenciadas en esta investigación documental permiten acentuar la naturaleza multi e interdisciplinar de la nanoquímica. Posteriormente, se discuten aspectos que permiten justificar su relevancia educativa. En la última parte de este manuscrito se ofrece una descripción de diversas propuestas desarrolladas en el bachillerato y en las etapas iniciales de la formación universitaria en torno a la enseñanza de contenidos que guardan relación con la nanoquímica, ya que estas pueden orientar y robustecer el trabajo pedagógico en este campo.

PALABRAS CLAVE: nanoquímica, enseñanza de la química, currículo de química, educación en nanotecnología, metodología bottom-up.

Recibido: 4 de mayo 2021.

Aceptado: 26 de julio 2021.

Publicado: 25 de agosto 2021.

* Universidad Nacional Autónoma de México, Colegio de Ciencias y Humanidades, México.

Correspondencia: jormeinguer@yahoo.com.mx 


\section{Introducción}

En las últimas décadas la química ha abierto nuevas rutas de investigación multidisciplinar al fusionar conocimientos de sus cinco áreas clásicas representadas por la química orgánica, la química inorgánica, la química teórica computacional, la química analítica y la fisicoquímica (Atkins, 2013). Un ejemplo de ello es la nanoquímica, un campo que permite sintetizar materiales o sistemas complejos mediante métodos basados en el autoensamblaje molecular (Alonso-Núñez, 2008). El prefijo nano es un derivado del latín nanus que significa enano en español y hace alusión a la milmillonésima parte de un metro $\left(1 \times 10^{-9} \mathrm{~m}\right)$. Para tener una idea más clara de lo diminuta que es la escala nanométrica, se pueden citar algunos ejemplos como el diámetro de un cabello humano de aproximadamente 75,000 nm, el de un glóbulo rojo de 3,000 nm y la distancia entre dos átomos de oro en un anillo o moneda de este elemento químico es de $0.3 \mathrm{~nm}$. En consecuencia, estudiar el mundo nano es situarse en el marco de acción de los átomos y las moléculas (Takeuchi, 2011). Un escenario afín para la química, al constituir un marco explicativo común en el estudio de las propiedades, la composición y la transformación de la materia.

La nanoquímica engloba aquellas actividades que utilizan las aproximaciones, herramientas y modelos tradicionales de la química para la obtención de materiales en escala nanométrica. Los productos nanoestructurados así obtenidos poseen diversas aplicaciones que van desde la catálisis y la obtención de fármacos de mayor especificidad hasta la producción de celdas fotovoltaicas, dispositivos ópticos, semiconductores y polímeros reforzados o biodegradables (Fasenko y Yatsenco, 2018).

En el ámbito escolar, la comunicación de aspectos relacionados con la nanoquímica se devela como una cuestión pertinente por tres razones: la primera es que, al ser un área de investigación reciente, su abordaje en las aulas y laboratorios permite dotar de actualidad el proceso de enseñanza y aprendizaje de la química; la segunda se relaciona con la posibilidad de emprender un proceso de enseñanza-aprendizaje del tema basado en la contextualización, al mostrar la efectividad que poseen las aplicaciones que se desprenden de la nanoquímica en la resolución efectiva de problemas que impactan la vida social, económica y ambiental, y, la tercera razón se vincula con promover en el estudiantado la construcción de una opinión informada sobre los beneficios, limitaciones y riesgos asociados con la nanoquímica en particular y con la nanotecnología en general, un requisito indispensable para promover la participación en el debate público sobre el tema.

Algunos especialistas señalan que promover el diálogo informado entre todos los sectores involucrados en el desarrollo de las nanociencias y de manera externa con la sociedad es la forma más loable y efectiva para que los diversos productos que genera este campo resulten beneficiosos para las mayorías y mantengan una armonía con el medio ambiente (Strand y Nydal, 2008; Delgado, 2011). Lo que se busca es la construcción de un entendimiento ge- 
nuino de la relevancia e implicaciones de las nanociencias por parte de la sociedad, el cual contribuya a robustecer sus criterios de gobernanza ${ }^{1}$ (Delgado, 2012). La educación formal e informal tienen un papel clave en la consecución de este objetivo, pues tanto las instituciones educativas como los medios divulgativos constituyen las vías principales para acercar los conocimientos y avances que genera la investigación nano a la sociedad. La formación de una ciudadanía crítica y participativa en materia de ciencia y tecnología es uno de los objetivos clave tanto de las sociedades democráticas como de los paradigmas educativos contemporáneos (Santos y Auler, 2019).

En los siguientes párrafos se abordarán algunos antecedentes históricos de la nanoquímica, sus conceptos básicos, su metodología, algunas aplicaciones representativas, así como su relevancia y presencia en el ámbito escolar.

\section{Antecedentes históricos}

Como primer referente de la nanoquímica se puede señalar una conferencia dictada por el connotado físico Richard Feynmann en 1959 en el Instituto Tecnológico de California (Caltech), titulada como There's plenty of rooom at the bottom (hay mucho espacio en el fondo). En esta charla académica Feynmann planteó la posibilidad de manipular la materia átomo por átomo para crear materiales con propiedades insólitas o poco conocidas (Feynmann, 1960). A su vez, marcó la pauta para la obtención de materiales por los dos enfoques más importantes que posee la nanotecnología, la metodología bottom-up (síntesis ascendente) tradicionalmente implementada por los químicos, la cual consiste en la construcción desde lo pequeño hacia lo grande y la metodología top-down (síntesis descendente) basada en la miniaturización que es convencionalmente usado por la física (Bensaude-Vincent, 2004). Cabe señalar que, aunque estos dos enfoques fueron desarrollados de forma independiente en la actualidad no rivalizan, sino que son complementarios. Un ejemplo de ello es su utilización conjunta tanto en cursos formativos como en investigaciones enmarcados en la ciencia e ingeniería de materiales.

Tuvieron que pasar 30 años para que las ideas de Feynmann sobre la posibilidad de incidir en el comportamiento de la materia en la escala nanométrica cristalizaran. Esto ocurrió en el año 1989, cuando científicos de la empresa IBM utilizando los avances de la época sobre microscopía de efecto túnel (STM), lograron obtener un arreglo de 35 átomos individuales de xenón en un sustrato de níquel con la forma de las tres letras del logo de dicha compañía. Este acontecimiento se convirtió en un hito para la ciencia moderna, pues por primera vez se conseguía colocar con precisión una cantidad de átomos determinada sobre una superficie plana (Bayda y Adeel, 2020).

${ }^{1}$ La gobernanza se entiende como un proceso configurado por una multiplicidad de reglas formales e informales que establecen las normas del juego entre los distintos actores (y niveles) que intervienen en el proceso de toma de decisiones (García, 2012). 
Mundo Nano | ARTículos DE INVESTIGACIÓN | www.mundonano.unam.mx 15(28), 1e-24e, enero-junio 2022 | https://doi.org/10.22201/ceiich.24485691e.2022.28.69674 Jorge Meinguer Ledesma

FIGURA 1. Logotipo de la compañía IBM obtenido en 1989, al colocar 35 átomos de xenón sobre un sustrato de nitrato de níquel mediante microscopía de efecto túnel (STM).
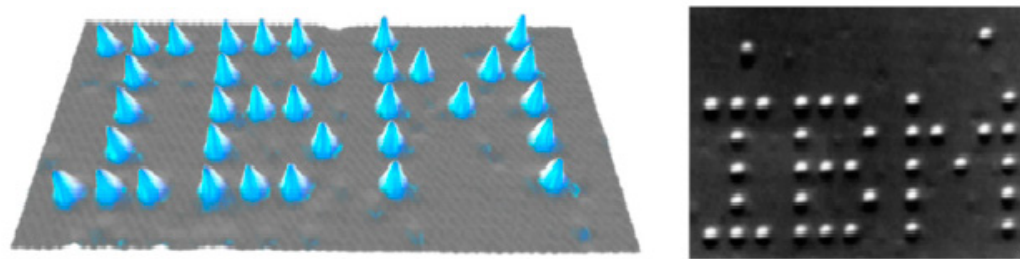

Fuente: Imagen tomada de Bayda y Adeel (2020).

En 1985, el descubrimiento que contribuyó a sentar las bases de la nanoquímica fue reportado por un equipo de investigación de la Universidad de Rice, conformado por los químicos Harold W. Croto, Robert F. Curl y Richard E. Smalley, quienes comunicarón el hallazgo de una molécula esférica de 60 átomos de carbono con una geometría muy similar a un balón de fútbol y de tan solo $0.7 \mathrm{~nm}$ de diámetro. La obtención de esta molécula hasta el momento desconocida se dio en el marco de un experimento consistente en vaporizar grafito con un láser a una temperatura superior a los $10,000{ }^{\circ} \mathrm{C}$ para simular agregados de carbono presentes en el medio interestelar. La molécula esférica resultante fue nombrada fullereno $C_{60}$ y es considerada el primer alótropo nanoestructurado reportado del elemento carbono. Por este trabajo, el equipo lidereado por Harold W. Croto se hizo merecedor del Premio Nobel de Química en el año 1996. En la actualidad, se han sintetizado fullerenos de varios tamaños, siendo el más pequeño el de 20 átomos de carbono $C_{20}$, pero el más abundante y representativo sigue siendo el $\mathrm{C}_{60}$ (Martín, 2011). La investiga-

FIGURA 2. Equipo de investigación que reportó el hallazgo de los fullerenos en 1985. De izquierda a derecha, Sean O־Brien, Richard Smalley, Robert Curl, Harold Kroto y James Heath.

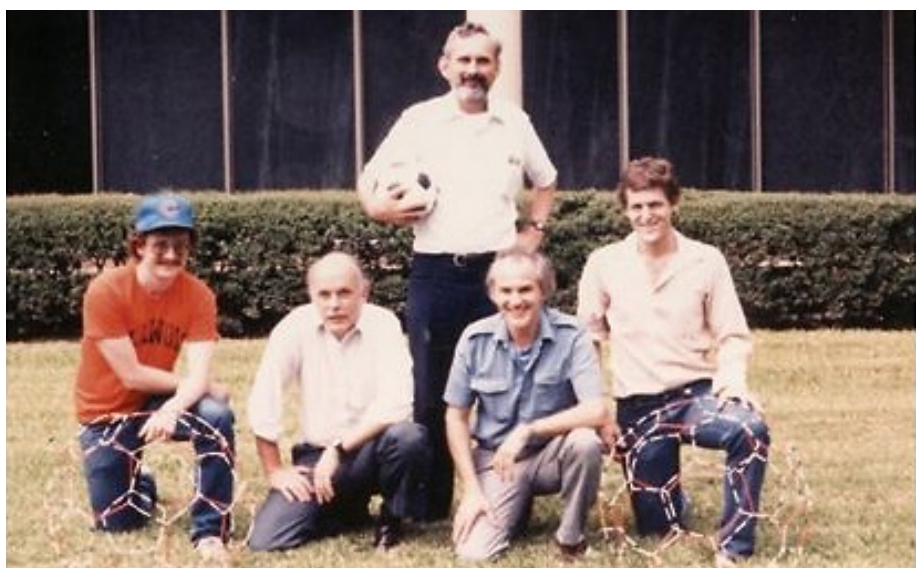

Fuente: Imagen tomada de (Martín, 2011). 
ción en torno a los fullerenos se extendió en años posteriores dando como resultado la obtención de unas moléculas cilíndricas denominadas nanotubos de carbono (NTC), nanoestructuras que se caracterizan por su alta resistencia.

El enfoque bottom-up en la obtención de nanoestructuras y nanomateriales fue popularizado lúcidamente por Richard E. Smalley al inicio del siglo XXI, gracias a su participación en un polémico y famoso debate sobre la forma de conceptualizar la nanotecnología que sostuvo con el ingeniero del MIT, Erick Drexler. Este comentado debate se llevó a cabo entre los años 2001 y 2003 en dos revistas de divulgación científica norteamericanas Scientific American y Chemical \& Engineering News, y ha sido estudiado arduamente por especialistas de las ciencias sociales para ilustrar el papel que detentan los medios de comunicación y aspectos para-científicos en la delimitación de una agenda en torno a campos tecnocientíficos emergentes (Kaplan y Radin, 2011). En el debate, R. Smalley comunicó con sagacidad los factores que la química toma en cuenta en la obtención de nanomateriales como es el caso del estudio de las condiciones de reacción, el uso de catalizadores, la consideración de la geometría, el tamaño, la reactividad y la selectividad de las especies reaccionantes, así como una variable muy importante resultante de los procesos de autoensamblaje molecular, las imperfecciones (Bueno, 2004). En contraposición de una visión basada en la obtención de algo que Drexler denominó ensambladores moleculares, una especie de máquinas nonoscópicas que permitirían el control posicional absoluto de átomos y moléculas en la obtención de nanomateriales. Con el pasar de los años esta visión de Drexler se develó errónea. De hecho, las imperfecciones defendidas por Smalley poseen un papel tan relevante en la síntesis de algunos materiales nanométricos que gracias a estas adquieren funciones específicas y distintivas. Un ejemplo de ello son los vidrios dopados con moléculas orgánicas obtenidos por el método sol-gel, sus propiedades ópticas y alta estabilidad térmica se deben en gran medida a la geometría imperfecta de la matriz vidriosa (Sebastián, 2018).

Otro antecedente importante de la nanoquímica es la denominada química supramolecular. En 1987, los químicos Donald J. Cram, Charles J. Pedersen y Jean-Marie Lehn, recibieron el Premio Nobel de Química por diseñar moléculas que imitan el comportamiento de algunas sustancias naturales implicadas en procesos biológicos. Estos investigadores demostraron que en los laboratorios se pueden obtener moléculas altamente selectivas que pueden reaccionar con otros átomos, tal como lo hacen las enzimas en los procesos celulares (Munuce, 2014). Para ello, implementaron técnicas avanzadas de química orgánica acompañadas de complejos cálculos teóricos. Los resultados reportados por el equipo de Donald Cram aumentaron el interés y el dominio de la selectividad en la obtención de complejos químicos. Hoy en día, la selectividad es un factor crucial en el autoensamblaje molecular, proceso en el que está fundamentada la nanoquímica. Finalmente, una investigación que puede considerarse también como un precedente histórico de la nanoquímica fue el descubrimiento de los polímeros conductores por los químicos Alan Hegger, 
Alan MacDiarmid y Hideki Shirakawa por el cual recibieron el Premio Nobel de Química en el año 2000 (Aguayo y Zarzuela, 2011).

\section{Generalidades de la nanoquímica}

La nanoquímica robustece el horizonte de investigación de la química al ocuparse de la síntesis y la caracterización de materiales desarrollados a través de técnicas o procedimientos basados en el autoensamblaje molecular (Contreras y Cardoso, 2015). Este fenómeno se basa en el aprovechamiento de fuerzas débiles o intermoleculares como las de Van der Waals, los puentes de hidrógeno y las interacciones electrostáticas que se dan entre las entidades químicas reaccionantes. Una cuestión que favorece la espontaneidad y cierto grado de control termodinámico es que, en el autoensamblaje, los átomos y las moléculas buscan adquirir un estado de mínima energía difundiéndose sobre superficies o formando estructuras que les favorezcan energéticamente y potencien el crecimiento molecular (Ozin y Arsenault, 2015).

Lo que hace particular e interesante tanto a la nanoquímica como a las nanociencias en general, es que en la escala nanométrica las propiedades de la materia cambian drásticamente debido a efectos cuánticos. Este inusitado comportamiento puede llegar a contradecir lo que percibimos con nuestros sentidos. Por ejemplo, cuando los metales como el oro, la plata y el cobre se dividen en diminutos fragmentos que rozan la escala nanométrica su brillo metálico desaparece paulatinamente dando lugar a tonalidades poco convencionales. Se sabe que las esferas de oro de 100 nanómetros (nm) adquieren una tonalidad naranja, las esferas de $50 \mathrm{~nm}$ son verdes y las de $25 \mathrm{~nm}$ son rojas. Este hecho se debe a que los electrones libres de conducción presentes en las superficies de las esferas metálicas de tamaño nanoscópico pueden oscilar por efecto de la luz y absorber energía (López-Quíntela, 2015). De lo anterior, se puede señalar que otra característica distintiva de la nanoquímica es que suele auxiliarse y aplicar las bases teóricas de la mecánica cuántica en la síntesis y caracterización de materiales.

Como se ha mencionado en párrafos anteriores, el enfoque que ha acompañado el desarrollo de la nanoquímica es el denominado bottom-up, el cual consiste en utilizar grupos de átomos y moléculas como precursores o bloques de construcción de estructuras de orden superior (Aguayo-González y Zarzuela, 2011). Las técnicas bottom-up se pueden clasificar en dos grandes categorías de acuerdo con el entorno físico en el que se obtienen los nanomateriales, estas son técnicas en fase gas y fase líquida (Sebastián, 2018). Ejemplos de técnicas bottom-up que se llevan a cabo en fase gaseosa son la deposición en vapor y la ablación láser. Mientras que en los métodos de fase líquida destacan las microemulsiones, la síntesis sol-gel y los basados en la fotoquímica (Ozin y Arsenault, 2015). Otro aspecto importante a considerar en la nanoquímica es que se auxilia de diversas técnicas de la microscopía moderna en la caracterización de las propiedades fisicoquímicas de los nanoma- 
teriales, siendo las más usuales la microscopía de efecto túnel (STM) y de barrido (SEM).

\section{Técnicas en fase gaseosa}

La deposición en vapor por vía química CVD (del inglés chemical vapor deposition) es una de las técnicas más representativas en fase gaseosa, se basa en la dispersión de un precursor químico en estado gaseoso sobre un sustrato donde se pretende llevar a cabo el crecimiento de un nano o micromaterial (Sebastián, 2018). Generalmente, los procesos de síntesis basados en CVD se llevan a cabo en cámaras de alto vacío, son altamente selectivos y se utilizan para la obtención de algunas nanoestructuras sólidas como el óxido de silicio $\left(\mathrm{SiO}_{2}\right)$ y los nanotubos de carbono (NTC). Por su parte, la técnica de ablación láser consiste en someter un blanco a una intensa radiación con ayuda de un láser con el objetivo de escindir en este sus constituyentes, para que posteriormente estas sustancias vuelvan a reaccionar formando entidades químicas diferentes, las cuales son denominadas como nanoclusters (Cruz-Alonso et al., 2018). La síntesis por ablación láser es un proceso menos selectivo que la CVD, pero es una técnica más limpia porque genera menor cantidad de residuos y es usualmente empleada para la manufactura de algunos superconductores de alta temperatura (Bäurle, 2013).

\section{Técnicas en fase líquida}

Las técnicas de fase líquida son las más utilizadas en la nanoquímica, al involucrar la acción de un disolvente y procedimientos relacionados con la cinética de cristalización de los productos deseados, dos aspectos metodológicos históricos y distintivos del trabajo experimental que emprenden los químicos. Otros elementos a considerar en las técnicas bottom-up en fase líquida es el uso de agentes reductores y estabilizadores. Los primeros aportan electrones en un medio de reacción, favoreciendo con ello, los procesos de autoensamblaje molecular. Los segundos suelen ser de gran utilidad en el control de la morfología de los nanomateriales (Contreras y Cardozo, 2015).

Las técnicas bottom-up en fase líquida son más numerosas que las implementadas en fase gas, por motivos de extensión; y dado que no es menester de este trabajo cubrir todas las técnicas a detalle, en los siguientes párrafos solamente se hará referencia a la síntesis por sol-gel, microemulsiones y vía fotoquímica. La técnica sol-gel es un proceso químico ampliamente utilizado en la obtención de nanomateriales cuya estructura principal son óxidos metálicos. Generalmente, se parte de una solución química (sol) que actúa como precursor de una red integrada de partículas o de una red de polímeros (Palma y Acuña, 2010; Zanella, 2012; Rojas, 2015). Los precursores químicos más utilizados en este método son los cloruros y los alcóxidos metálicos, compuestos que en solución experimentan reacciones de condensación y polimerización para dar lugar a una red tridimensional en forma coloidal (gel). El gel resultante se deshidrata paulatinamente o en ocasiones se calcina para obtener los 
productos deseados (De Jong, 2009). Con esta técnica se pueden sintetizar nanomateriales de alta pureza y homogeneidad. Ejemplo de nanomateriales que se producen por sol-gel son combinaciones de óxidos de silicio, titanio y vanadio que al funcionalizarlos con iones de tierras raras pueden ser utilizados para la fabricación de láseres y sensores (Rojas, 2015).

Por su parte, las microemulsiones son dispersiones termodinámicamente estables formadas por líquidos inmiscibles de diferente polaridad (por ejemplo, agua/aceite). Las microemulsiones convencionalmente son estabilizadas por la adición de un surfactante o tensoactivo que permite la formación de micelas con un rango de tamaño de 2 a $15 \mathrm{~nm}$ (Aegerter et al., 2008; Zanella, 2012). En esta técnica, las micelas son las encargadas tanto de difundir los precursores químicos como de propiciar el crecimiento molecular. Algunas características importantes de las microemulsiones son su isotropía, transparencia y que a diferencia de las emulsiones macroscópicas no requieren de un aporte energético para su formación (Sebastían, 2018). Además, con esta técnica es posible controlar las dimensiones, la morfología y la composición de los productos sintetizados. Ejemplos del tipo de nanoestructuras que se pueden obtener con microemulsiones son las partículas binarias de elementos metálicos ( $\mathrm{Pt} / \mathrm{Pd}$, $\mathrm{Pt} / \mathrm{Ru}, \mathrm{Pt} / \mathrm{I}$ ), así como nanopartículas de óxidos y sulfuros metálicos (Takeuchi, 2011). Esta técnica también es utilizada en la elaboración de nanomateriales con repercusiones en la industria de los fármacos y los cosméticos.

Por su parte, la obtención de nanoestructuras basada en la fotoquímica se fundamenta en la irradiación de un medio de reacción con haces de luz de diferentes longitudes de onda. Esto con la finalidad de generar especies reductoras altamente reactivas como electrones, radicales o especies oxidadas que favorezcan la formación de nanoestructuras (Ozin y Arsenault, 2015). En las técnicas fotoquímicas usualmente se utilizan energías por debajo de los 60 $\mathrm{eV}$, se caracterizan por ser procesos rápidos y limpios que generan agregados

FIGURA 3. Izquierda: imagen obtenida por microscopía electrónica de transmisión (TEM) de nanopartículas de rutenio sintetizadas por la técnica de microemulsiones. Derecha: imagen obtenida con microscopía electrónica de barrido (STM) de esferas de óxido de silicio producidas con el método sol-gel.

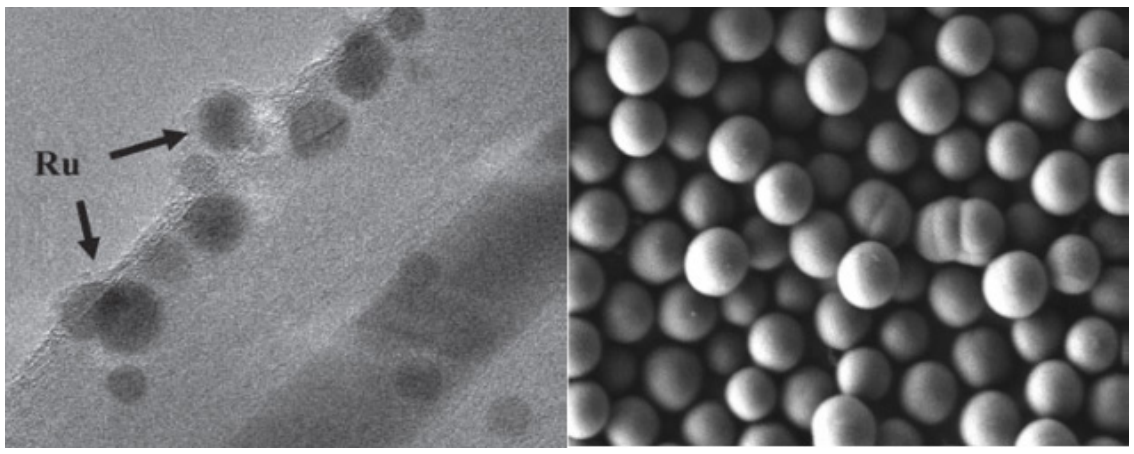

Fuente: Imagen izquierda tomada de Alonso-Núñez (2008); derecha, de Zanella (2012). 
moleculares sólidos (Zanella, 2012). Los métodos fotoquímicos son utilizados en la obtención de materiales con metales nobles, los cuales pueden ser empleados como fotocatalizadores y en la elaboración de dispositivos ópticos (Névarez-Martínez y Espinoza-Montero, 2017).

\section{Microscopía}

Un elemento de gran importancia en la nanoquímica es la microscopía, una ruta instrumental convencionalmente utilizada para caracterizar las propiedades estructurales y fisicoquímicas de los nanomateriales sintetizados. Las técnicas microscópicas de las que se apoya la química y la nanotecnología consisten, en general, en someter un material o blanco a un tipo de radiación específica por medio de una sonda y decodificar las señales subyacentes mediante complejos sistemas de detección, los cuales pueden estar basados en las interacciones que se dan entre los electrones presentes en la muestra y los de la sonda o en la medición de propiedades físicas específicas como el voltaje, la intensidad de corriente y la intensidad de un campo magnético (Takeuchi, 2011; Contreras y Cardozo, 2015). Con ayuda de la microscopía, se pueden obtener de manera indirecta imágenes y datos relevantes sobre las propiedades de nanoestructuras específicas.

FIGURA 4. Imágenes de nanotubos de carbono (NTC) obtenidas con microscopía electrónica de barrido (SEM).

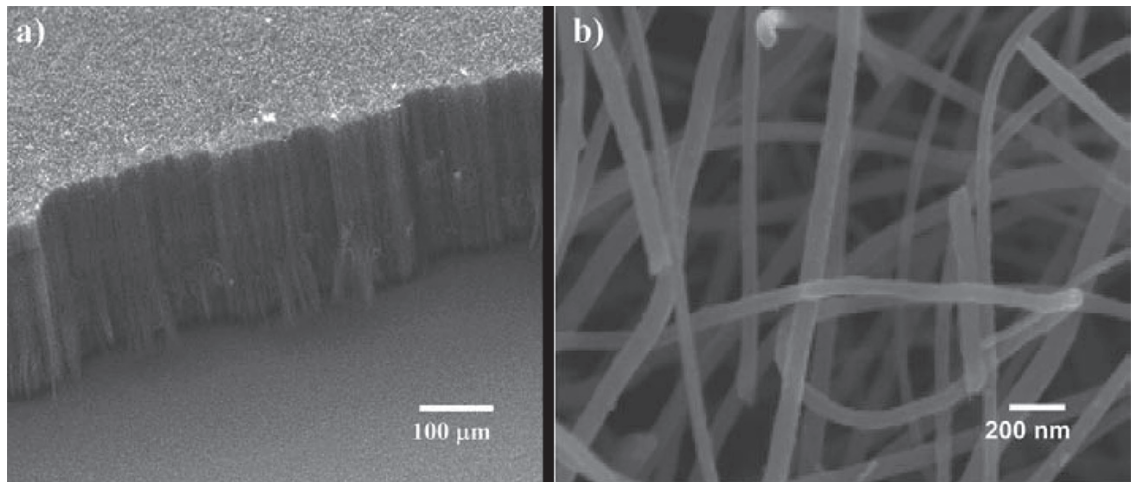

Fuente: Tomadas de Alonso-Nuñez (2008).

Las técnicas microscópicas más usuales en la nanoquímica y que han estado presente en su desarrollo histórico son la microscopía de efecto túnel (STM), la microscopía electrónica de transmisión (TEM) y la microscopía electrónica de barrido (SEM). En los últimos años y en menor medida, algunas investigaciones han incorporado también el uso de la microscopía de fuerza atómica (AFM), esto debido a la importancia tecnológica que ha cobrado el estudio de las superficies en el campo de los nanomateriales (Contreras y Cardozo, 2015). 


\section{Aplicaciones e implicaciones socioambientales de la nanoquímica}

En las últimas décadas tanto la nanoquímica como la nanotecnología han ampliado significativamente y diversificado sus campo de aplicaciones. Los materiales que está generando la química en la escala nanométrica tienen aplicaciones en el campo de la catálisis, los polímeros, los medicamentos, la fabricación de recubrimientos, dispositivos ópticos y fotónicos, en la elaboración de fragancias y aditivos alimenticios, así como en la biotecnología y la remediación ambiental (Pagliaro, 2015). En los siguientes párrafos se describen algunas aplicaciones de la nanoquímica en el ámbito de la catálisis, la síntesis de polímeros y la ciencia de materiales. Se ha determinado referenciar únicamente estos tres nichos de aplicación porque constituyen áreas representativas del conocimiento químico y por las características de este trabajo.

Un catalizador es una sustancia que incrementa la rapidez de una reacción química, esto al modificar su cinética y generar un mecanismo de reacción alterno con una energía de activación menor (Díaz, 2011). En la escala nanométrica destaca el campo de la catálisis heterogénea, es decir, sistemas en donde los catalizadores se dispersan en materiales altamente porosos. La disminución en el tamaño de partícula en los catalizadores permite que su superficie quede más expuesta, de tal modo que una alta cantidad de átomos presentes en esta se conviertan en sitios activos, incrementando la eficiencia de una reacción. Ejemplos de catalizadores nano son los agregados moleculares de óxidos y sulfuros metálicos producidos por las técnicas sol-gel. Se ha reportado que partículas de óxido de titanio $\left(\mathrm{TiO}_{2}\right)$ favorecen algunos procesos de biorremediación ambiental, como es el caso de la adsorción del dióxido de carbono $\left(\mathrm{CO}_{2}\right)$ y ácido sulfúrico $\left(\mathrm{H}_{2} \mathrm{SO}_{4}\right)$, dos gases contaminantes presentes en la atmósfera. $\mathrm{El} \mathrm{TiO}_{2}$ como catalizador también es referenciado en algunos métodos recientes que guardan relación con la purificación del agua (Escobar y Solís-Casados, 2020). En la literatura se menciona que algunos nanocatalizadores hechos a base de sulfuros de molibdeno con trazas de níquel $(\mathrm{Ni})$ y cobalto $(\mathrm{Co})$ soportados en alumina $\left(\mathrm{Al}_{2} \mathrm{O}_{3}\right)$ optimizan la remoción de los compuestos orgánicos de azufre (COA) en diferentes fracciones del petróleo (Álvarez-Amparán, Cedeño-Caero, 2020). Otro grupo de catalizadores nanoestructurados por destacar son los realizados a base de paladio (Pd) y soportados en materiales aislantes o semiconductores que al ser dopados con tierras raras resultan eficientes en la reducción de $\mathrm{CO}$ e hidrocarburos (Díaz, 2011). Actualmente, más del 90\% de los procesos químicos en el mundo se auxilian de la catálisis y los nanocatilizadores ocupan un lugar importante.

En el ámbito de los polímeros, la nanoquímica está contribuyendo a mejorar las propiedades de estos materiales incorporando nanoestructuras específicas. Por ejemplo, cuando se adicionan nanopartículas de sílice a polímeros termoplásticos como el poliuretano se mejoran sus propiedades mecánicas, 
térmicas y de adhesión (Martín y Vega, 2013). En la literatura se menciona que, al enriquecer materiales como el acrílico con nanopartículas de cobre se incrementa su comportamiento antibacteriano, un hecho relevante en el ámbito biomédico porque el acrílico termopolimerizable (PMMA) es muy utilizado en la elaboración de prótesis de diversos tipos (Camacho y Arenas, 2015). Otro tipo de materiales provistos por la nanoquímica y de amplia repercusión en la industria de los polímeros son las nanofibras, esto es, fibras con diámetros de hasta $100 \mathrm{~nm}$ que pueden ser de origen natural o sintético. Este tipo de fibras se suelen producir por el método de electrohilado y mejoran la calidad de materiales ya existentes debido a su reducida área superficial. Actualmente, se utilizan nanofibras para la elaboración de ropa de protección y deportiva, en materiales que permiten la liberación controlada de medicamentos, soportes para el crecimiento celular y en la elaboración de membranas poliméricas (García, 2013).

Otra línea de investigación representativa de la nanoquímica es la relacionada con la obtención de materiales cuya base estructural son los alótropos nanoestructurados del elemento carbono, como es el caso de los fullerenos, nanotubos de carbono (NTC) y el grafeno. En las siguientes líneas se hará referencia a algunas aplicaciones basadas en los dos primeros, pues la investigación química en torno al grafeno es relativamente reciente y se encuentra en proceso de consolidación. Las aplicaciones más citadas de los fulleros son tres: 1) se suelen utilizar para fabricar lubricantes en la industria automotriz; 2) constituir un componente importante de células fotovoltaicas orgánicas, y, 3) poder ser empleadas como nanoestructuras inhibidoras de algunos virus.

En los lubricantes, las moléculas $C_{60}$ actúan como nanorrodamientos interponiéndose con las partes metálicas de los motores, reduciendo la fricción y aumentando su potencia (Takeuchi, 2011). En los paneles solares, los fullerenos son incorporados con el propósito de reducir la concentración de silicio y optimizar el funcionamiento de estos dispositivos. En este tipo de células fotovoltaicas, un polímero orgánico se encarga de absorber la luz solar y provocar la excitación de los electrones, los fullerenos por sus propiedades semiconductoras actúan como aceptores, favoreciendo el flujo de estas partículas en estos dispositivos (Bolaños y Álvarez, 2018). En el ámbito de la biomedicina, los fullerenos pueden ser útiles para administrar el principio activo de medicamentos de alta especificidad, esto por su alta capacidad de unirse a las proteínas y moléculas complejas. Concretamente, se ha reportado que derivados del fullereno $C_{60}$ tienen la propiedad de inhibir el desarrollo de algunos virus como el VIH y disminuir su propagación (Castro et al., 2017). En lo concerniente a los nanotubos de carbono destaca su utilización en el refuerzo de materiales poliméricos y en diversos procesos basados en la funcionalización química. Por ejemplo, cuando se adicionan NTC a polímeros tradicionales, estos mejoran sus propiedades mecánicas, térmicas, su resistencia, durabilidad e incluso en algunos casos les confieren la propiedad de conductividad 
(Isaza et al., 2019). Por otra parte, cuando los NTC son funcionalizados, esto es, cuando se incorporan a su estructura diversos grupos funcionales o se dopan con metales, se obtienen nanomateriales híbridos útiles en la fabricación de biosensores, baterías y materiales de la industria aeroespacial (Kaurosis et al., 2010). El campo de los nanomateriales hechos a base de carbono es muy versátil y ha generado una gran cantidad de publicaciones científicas en la última década.

Al ser la nanoquímica un área de investigación emergente tiene asociada una fuerte carga de incertidumbre, relacionada principalmente con la toxicidad y el impacto ambiental de los materiales que está generando para el ramo de la industria y la investigación. Es por ello que filósofos, sociólogos, economistas, educadores y divulgadores aportan contribuciones valiosas en lo relativo al análisis ético, económico, socioambiental y educativo de la investigación nano, reforzando su dimensión interdisciplinar (Foladori e Invernizzi, 2018). Algunas acciones que están planteando estos expertos para aminorar los riesgos de las nanociencias y la nanotecnología son la implementación de protocolos de ensayo de las nanopartículas de origen sintético, el establecimiento de normas que permitan regular sus repercusiones ambientales y en materia de sanidad, el estudio de su relevancia e implicaciones en la educación científica formal e informal, así como una serie de recomendaciones en materia de gobernanza de la nanotecnología (Pyrrho y Schramm, 2019).

Por lo anterior, se puede aseverar que la nanoquímica en particular y la nanotecnología en general poseen un rostro jánico, al poder contribuir significativamente en el bienestar humano, al potenciar el desarrollo tecnológico y sostenible. Sin embargo, si sus avances no cuentan con una regularización basada en la ética, el cuidado del medio ambiente y el bienestar común, así como sus desarrollos pueden ser desvirtuados y generar enfermedades, agudizar problemáticas ambientales, conflictos sociales y económicos producto de la sobrexplotación de recursos y su posterior comercialización. Esta dualidad es una cuestión muy importante a tener en cuenta en la labor educativa en lo que respecta a la nanoquímica.

\section{Relevancia y problemas asociados con la enseñanza de la nanoquímica}

Como se ha señalado en la introducción de este trabajo, la incorporación de asignaturas y contenidos relacionados con las nanociencias y la nanotecnología en los diferentes niveles educativos puede redituar en un entendimiento más profundo y reflexivo tanto de la relevancia como de las implicaciones tecnológicas, sociales y ambientales de este campo de conocimientos. Los procesos de diálogo social y la toma de decisiones informadas (Delgado y Magaña, 2012) son elementos que forman parte de lo que en la literatura se ha denominado como gobernanza precautoria de la nanotecnología, esto es, una serie de criterios que permiten establecer una política pública e incluyente en ma- 
teria de nanociencia y nanotecnología que garantice la seguridad, el desarrollo social, la innovación y la preservación ambiental (Saldívar, 2021). En este contexto, las universidades e instituciones de educación obligatoria juegan un papel importante en lo relativo a garantizar la inclusión social, establecer códigos de conducta basados en el conocimiento y fortalecer los mecanismos de participación en los procesos de diálogo social vinculados con la investigación nano.

Otras razones que justifican el impulso del trabajo educativo en torno a la nanoquímica en los niveles medio y superior son la necesidad de formar vocaciones científicas en el campo, ofrecer una visión correcta y equilibrada de esta subdisciplina, así como dotar de actualidad al proceso de enseñanzaaprendizaje de la química. En el contexto actual, las profesiones, áreas de investigación y empleos relacionados con la síntesis de materiales en la escala nanométrica se han incrementado y diversificado notablemente, resultando oportuno promover en el ámbito escolar el interés por esta área de investigación, para que, paulatinamente, un mayor número de estudiantes decidan especializarse (Plagiario, 2015). Asimismo, promover una visión correcta y más prudente de la nanoquímica y de la nanotecnología en general obedece a haber constatado que tanto en los medios de comunicación como en las redes sociales prevalece una imagen de la investigación nano errónea o deformada, al asociársela con metas ficticias, aplicaciones con una alta carga especulativa y una retórica falaz proveniente de la pseudociencia. Cuestiones que distan mucho de la realidad imperante en los laboratorios y centros de investigación. Para hacer frente a este problema es necesario comunicar en las aulas y los laboratorios un panorama de la nanoquímica que parta de aquellos avances o aplicaciones que cuenten con un amplio respaldo entre la comunidad de especialistas.

La labor educativa en torno a la nanoquímica permite referenciar aspectos novedosos relacionados con los procesos de síntesis, conectarlos con el estudio de otros contenidos donde la innovación adquiera valor, así como involucrar a los estudiantes con tres rasgos que son distintivos de la ciencia contemporánea: la interdisciplinariedad, la incertidumbre y la complejidad (Meinguer, 2019). Los procesos de síntesis química en la escala nanométrica requieren de una visión metodológica que articule modelos, herramientas y aproximaciones de las áreas clásicas de la química, así como elementos teóricos e instrumentales provenientes de otras disciplinas científicas. No obstante, es importante enfatizar en la labor educativa sobre el tema que, la dimensión multi e interdisciplinar de la nanoquímica no se agota en la construcción de un marco teórico-metodológico robusto e innovador, sino debe contemplar también la influencia de diversas disciplinas sociales al analizar el impacto que tienen sus desarrollos en la esfera de lo económico, ético, social, ambiental y cultural. Esto último conecta con el segundo rasgo que se postula como distintivo de la ciencia contemporánea: la incertidumbre. En el caso concreto de la investigación nano, la incertidumbre se relaciona con los efectos negativos que sus apli- 
caciones pueden generar en diversos escenarios, pero también con la posibilidad que tiene la ciencia y los demás sectores involucrados en corregirlos. La interdependencia disciplinar presente en la visión teórica-metodológica de la nanoquímica y la tensión derivada de promover innovaciones cuyos efectos no están completamente determinados y regulados, hace que esta área del conocimiento químico adquiera una alta complejidad. Por lo anterior, se puede afirmar que, diseñar e implementar estrategias basadas en el mundo de lo nano es una labor difícil de emprender, al requerir impulsar una formación que vaya más allá del dominio disciplinar de un tema.

En la literatura se han reportado algunos obstáculos epistemológicos que se deben considerar en el ámbito de la enseñanza y divulgación de las nanociencias. Los más importantes son:

- El manejo de una escala sumamente pequeña.

- La ineludible presencia de conceptos abstractos.

- El carácter multi e interdisciplinar de las nanociencias y la nanotecnología.

- La existencia de efectos vinculados con el tamaño y forma de los objetos.

- La existencia de ideas preconcebidas sobre la investigación nano (Sánchez-Mora y Tagueña, 2011).

Para atenuar estos obstáculos epistemológicos, en la literatura se recomienda hacer uso de un discurso analógico al comunicar conceptos complejos y relacionarlos con aspectos de la vida cotidiana, así como referenciar sustancias y organismos que resulten familiares al estudiantado. Se sugiere comunicar el tamaño y las funciones de los virus y moléculas como la del ADN, la penicilina o la glucosa para situar y despertar el interés por el comportamiento de la materia en la escala nanométrica (Takeuchi, 2011). Otras cuestiones relevantes a considerar en el proceso de enseñanza-aprendizaje de la nanoquímica son informar sobre los avances conseguidos a nivel local, nacional e internacional, incorporar el uso de las tecnologías de la información y comunicación (TIC) para modelar estructuras, materiales y dispositivos que tienen su origen en la escala nanométrica, así como crear escenarios de aprendizaje donde se propicie el debate racional de ideas, la indagación y la argumentación escolar (Garcia-Betancourt, 2017). En el primer caso, el análisis de fuentes provenientes de la comunicación pública de la ciencia como complemento de los libros de texto y la organización de charlas divulgativas pueden ser de gran utilidad, al ser rutas que permiten ofrecer información científica de forma precisa, clara, respaldada y contextualizada, resultando asequible para estudiantes de niveles medios y de la etapa inicial en universidades (Meinguer, 2018). En lo que respecta al uso de la TIC, los softwares, animaciones digitales y otro tipo de herramientas digitales resultan esenciales para modelar la estructura, los enlaces, propiedades, la cinética de las reacciones, 
los procesos de autoensamblaje y el comportamiento de las nanoestructuras y materiales obtenidos con la metodología bottom-up. Las TIC se consideran un elemento imprescindible y distintivo tanto en el trabajo metodológico como educativo de las nanociencias (Ribeiro y de Souza-Filho, 2014).

En el plano didáctico, es necesario diseñar actividades que contribuyan al desarrollo de habilidades teóricas, experimentales, de análisis, comunicación y participación, debido a que se ha mencionado que las áreas de oportunidad para los alumnos especializados en nanoquímica u otras áreas relacionadas con esta subdisciplina trascienden los ámbitos de la investigación y la enseñanza (Reviglio, 2014). Fomentar el pensamiento crítico: la indagación y la participación en la educación nano es apostar por una formación integral, una meta educativa que es ampliamente reconocida en todas las áreas de conocimiento científico (Meinguer, 2021).

\section{La nanoquímica en el currículum escolar}

El avance de la nanoquímica en la educación ha sido más lento y limitado de lo conseguido en el ámbito de la investigación científica, tecnológica e industrial, pues aunque la mayoría de los países cuenta con especializaciones y grados universitarios que certifican los estudios en esta área de conocimiento, en la educación química que se imparte en los niveles obligatorios no suelen figurar contenidos relacionados con la investigación nano o son muy escasos, debido a que se les otorga poca relevancia en los programas de estudio (Pagliario, 2015). A pesar de ello, Jones y Blonder (2013) señalan que los enfoques para abordar contenidos relacionados con las nanociencias y la nanotecnología son muy diversos, sobre todo en las etapas iniciales de algunas carreras o especializaciones de corte científico en las universidades. Para estos autores, la educación nano requiere para su consolidación el establecimiento y validación de estándares tanto de aprendizaje como de evaluación. Al respecto, en la literatura se menciona que, en países de Europa y en EUA se están consolidando cursos intensivos en las etapas de pregrado para ahondar en aspectos relacionados con la nanoquímica. Estos cursos suelen tener una duración de cuatro a ocho semanas y se imparten a estudiantes que poseen conocimientos suficientes sobre química, física, matemáticas y biología (Garcia-Betancourt, 2017; Pagliario, 2015). Un ejemplo es la propuesta del químico Geoffrey Ozin de la Universidad de Toronto, quién ha diseñado e implementado un curso de nanoquímica basado en la impartición de doce conferencias, las seis primeras son dictadas por connotados especialistas y abordan en su mayoría aspectos introductorios en el campo. Mientras que, las seis restantes son preparadas por los estudiantes con apoyo de un amplio material bibliográfico. El curso cierra con un espacio de discusión sobre propuestas innovadoras elaboradas por los alumnos sobre la síntesis, aplicación o regulación de nanomateriales. Esto con el propósito de incentivar el pensamiento creativo (Ozin y Cademartiri, 2011). 
En Palermo, el investigador Mario Pagliario (2015) ha diseñado un curso de nanoquímica centrado en comunicar aspectos relevantes y novedosos sobre la síntesis de materiales nanoestructurados, instrumentación e implicaciones socioambientales. El curso tiene una duración de 180 horas y está dividido en seis semanas, inicia con la lectura y el análisis de contenidos relacionados con tres nanomateriales específicos en bibliografía especializada. Posteriormente, hay un módulo exclusivo para el trabajo en el laboratorio y finaliza con la elaboración de un artículo científico por los alumnos. Los autores de los libros de texto consultados en el curso son investigadores con amplia experiencia y trayectoria en la síntesis de nanomateriales (tabla 1). Por su parte, el trabajo de laboratorio está enfocado en el desarrollo de habilidades básicas en la síntesis de los nanomateriales estudiados previamente en la bibliografía. Por último, con la elaboración de un artículo científico se busca que los estudiantes sean capaces de articular conocimientos disciplinares y contextuales sobre los nanomateriales en cuestión, así como potenciar el desarrollo de habilidades de escritura y comunicación sobre la nanoquímica. Los contenidos, actividades y bibliografía del curso de Mario Pagliario se muestran en la tabla 1.

TABLA 1. Descripción del curso Nanoquímica, materiales funcionales y soluciones a problemas globales.

\begin{tabular}{|c|c|c|c|}
\hline Contenido & Duración & Actividades & Material bibliográfico \\
\hline $\begin{array}{l}\text { 1. ¿Por qué lo nano es } \\
\text { diferente? Fundamentos de } \\
\text { las nanociencias y la } \\
\text { nanotecnología. }\end{array}$ & 6 horas & Tres lecturas & $\begin{array}{l}\text { L. Cademartiri, G. A. Ozin, } \\
\text { Concepts of nanochemistry, } \\
\text { Wiley-VCH, } 2009 .\end{array}$ \\
\hline $\begin{array}{l}\text { 2. La ruta nanoquímica y } \\
\text { nanomateriales básicos. } \\
\text { Avances en los métodos de } \\
\text { síntesis y ensamblaje de } \\
\text { nanomateriales. }\end{array}$ & 6 horas & Tres lecturas & $\begin{array}{l}\text { L. Cademartiri, G. A. Ozin, } \\
\text { Concepts of nanochemistry, } \\
\text { Wiley-VCH, } 2009 .\end{array}$ \\
\hline $\begin{array}{l}\text { 3. Nanomateriales porosos, } \\
\text { poliméricos y biomiméticos. }\end{array}$ & $\begin{array}{l}12 \text { horas } \\
\text { (módulo doble) }\end{array}$ & Seis lecturas & $\begin{array}{l}\text { G. Cao, Nanostructures and } \\
\text { nanomaterials: synthesis, } \\
\text { properties and applications, } \\
\text { Imperial College Press, } \\
\text { Londres, } 2004 .\end{array}$ \\
\hline $\begin{array}{l}\text { 4. Nanomateriales en acción. } \\
\text { Almacenamiento de energía, } \\
\text { catálisis y recubrimientos. }\end{array}$ & 6 horas & $\begin{array}{l}\text { Tres sesiones } \\
\text { de } \\
\text { capacitación } \\
\text { en el } \\
\text { laboratorio }\end{array}$ & $\begin{array}{l}\text { M. Pagliaro, Nano-age: how } \\
\text { nanotechnology changes our } \\
\text { future, Wiley-VCH, } 2010 \text {. }\end{array}$ \\
\hline $\begin{array}{l}\text { 5. Aprendizaje permanente y } \\
\text { comunicación efectiva de la } \\
\text { nanoquímica y la } \\
\text { nanotecnología. }\end{array}$ & 6 horas & Tres lecturas & $\begin{array}{l}\text { M. Tomczyk, Nano } \\
\text { innovation: what every } \\
\text { manager needs to know, } \\
\text { Wiley-VCH, } 2014 \text {. } \\
\\
\text { C. Qian, T. Siler, G. A. Ozin, } \\
\text { Exploring the possibilities and } \\
\text { limitations of a nanomaterials } \\
\text { genome: small, 2015, 11, } 64 \text {. }\end{array}$ \\
\hline
\end{tabular}

Fuente: Traducido de Pagliario (2015). 
En el contexto iberoamericano, específicamente en el bachillerato destaca la guía didáctica elaborada por los académicos Pedro Serena, Noboru Takeuchi y Javier Tutor (2014). En este recurso educativo se ofrece una cantidad considerable de fichas didácticas que los docentes pueden consultar al abordar diferentes aspectos sobre las nanociencias y la nanotecnología, como es el caso de sus fundamentos teóricos, cuestiones generales sobre microscopía electrónica, la dimensión y efectos asociados con nanomateriales entre otros. En el caso concreto de la nanoquímica, se puede encontrar material sobre la síntesis de partículas de plata, nanomateriales basados en óxidos metálicos, nanocosméticos, nanosensores e implicaciones éticas y sociales vinculadas con el mundo de lo nano. La ventaja de esta publicación educativa es que las propuestas de tratamiento de los temas antes mencionados se pueden readaptar o adecuar a los objetivos de aprendizaje de un docente o una institución educativa.

Una metodología alternativa es la reportada por Meinguer (2019), en esta se analizan la estructura, propiedades y aplicaciones de los alótropos nanoestructurados del carbono (fullerenos, nanotubos de carbono y grafeno) mediante la lectura, análisis y problematización de textos provenientes de la comunicación pública de la ciencia. El trabajo educativo de esta propuesta gira en torno al diseño de estrategias de análisis textual que contemplen la realización de investigaciones documentales y escenarios de debate con el propósito de que los estudiantes puedan articular aprendizajes disciplinares, contextuales y sobre la naturaleza de la ciencia que les permitan construir una interpretación amplia y crítica del tema. Por otra parte, Ribeiro et al., 2018 informan que el uso de la halografía y la realidad virtual en contextos escolares contribuye a superar barreras sensitivas e intuitivas al modelar la estructura química de materiales y al referenciar conceptos abstractos ligados con las nanociencias. Estos autores citan que este tipo de tecnología favorece la interactividad, el diálogo, la participación y la construcción de aprendizajes significativos.

En resumen, las propuestas educativas descritas con anterioridad sugieren abordar la nanoquímica como una cuestión sociocientífica, es decir, como un campo de conocimientos con fuertes implicaciones sociales, tecnológicas, éticas y ambientales. Para ello, se debe tener en consideración los siguientes puntos: i) fomentar el dominio de los contenidos disciplinares en los que se fundamenta la nanoquímica, ii) promover el análisis de los riesgos e implicaciones socioambientales de los productos y desarrollos que tienen su origen en esta línea de investigación, y, iii) impulsar el pensamiento creativo, crítico y la innovación. Quedan aspectos adicionales por considerar como robustecer la formación de los profesores en esta área del conocimiento químico (sobre todo en el nivel bachillerato) y que los contenidos relacionados con la nanoquímica sean contemplados como relevantes por los especialistas e instituciones responsables de las reformas curriculares en materia de educación química. 


\section{La enseñanza de la nanoquímica bajo en el enfoque CTS-A}

Tomando en cuenta los cursos y estrategias referenciadas en los párrafos anteriores, se propone que una forma de encauzar el abordaje de la nanoquímica en el nivel medio y superior es a través del enfoque ciencia, tecnología, sociedad y ambiente (CTS-A), ${ }^{2}$ al constituir un marco educativo idóneo para dotar de coherencia y sentido los contenidos implicados. En el dominio de lo científico se pueden presentar los fundamentos que permiten explicar el comportamiento de la materia en la escala nanométrica y cómo las técnicas de síntesis agrupadas en la categoría bottom-up están modificando sustancialmente aspectos metodológicos e instrumentales de la química. En el rubro de lo tecnológico se sugiere comunicar cuestiones relacionadas con la amplia gama de aplicaciones que cubre la nanoquímica y aspectos relacionados con los sofisticados métodos espectroscópicos utilizados en la caracterización de nanomateriales. En este segundo apartado se recomienda incentivar el trabajo experimental, pues representa una parte sustancial en la enseñanza de todas las áreas de conocimiento químico. En el ámbito de lo social es posible abordar contenidos relacionados con el financiamiento, comercialización y gobernanza de la investigación nano. Finalmente, un elemento muy importante y al que no se le otorga la relevancia debida en las propuestas referenciadas con anterioridad es lo relacionado con la dimensión ambiental de la nanoquímica. Para cubrir este apartado, se propone, además, referenciar riesgos toxicológicos, diseñar actividades e instrumentos de evaluación basados en los diecisiete principios que la Organización de las Naciones Unidas asocia con el desarrollo sostenible (ONU, 2019). Esto con el objetivo de que los alumnos puedan indagar y valorar sobre qué tan sostenibles son las contribuciones que genera la nanoquímica.

La estructura de un curso de nanoquímica enraizado en el enfoque CTS-A puede estar organizado en cuatro módulos y culminar con la elaboración de proyectos de investigación encaminados a construir posibles soluciones a desafíos tecnológicos y ambientales en el ámbito local. Los módulos temáticos de esta propuesta son:

- Módulo 1. Introducción a la nanoquímica, conceptos y técnicas implicadas en la síntesis de nanomateriales.

- Módulo 2. Técnicas espectroscópicas y aplicaciones de la nanoquímica

- Módulo 3. Gobernanza de la nanoquímica y contribuciones al desarrollo sostenible

- Módulo 4. Desarrollo de proyectos de investigación y divulgación en nanoquímica

${ }^{2}$ El enfoque CTS-A es un movimiento educativo que surgió en la década de los años setenta y se consolidó a principios del presente siglo cuando aspectos relacionados con la historia, 
Como en los cursos descritos con anterioridad, se sugiere su impartición a alumnos que posean conocimientos elementales sobre química, física y naturaleza de la ciencia. En el contexto mexicano podría ser impartido después de que los alumnos completen con éxito lo que se denomina tronco común en los centros y facultades donde se imparten licenciaturas afines con la química. El curso puede tener una duración de ocho o diez semanas, siendo los módulos de mayor duración el 1 y el 4 (los relacionados con el abordaje del marco conceptual de la nanoquímica y el desarrollo de proyectos de investigación). El nivel de profundidad de cada tema dependerá de los objetivos de aprendizaje y el perfil profesional que delimiten las instituciones educativas.

En el bachillerato se propone condensar y adecuar el contenido de los cuatro módulos al nivel cognitivo que revisten los estudiantes en este nivel de estudios. Asimismo, se sugiere que el abordaje de la nanoquímica se dé en el cierre de las asignaturas de química que tienen carácter propedéutico, es decir, que se imparten en las últimas etapas del bachillerato a estudiantes cuyo perfil vocacional está orientado hacia la química o las disciplinas científicas. El tiempo por módulo puede ajustarse a una semana, se recomienda sustituir el trabajo experimental y los proyectos de investigación por actividades más asequibles para los estudiantes como reportes de visitas guiadas a museos o centros de investigación donde la nanoquímica ocupe un lugar relevante, la elaboración de escritos cortos como reseñas o ensayos que contribuyan a enriquecer el periodismos científico en las instituciones de nivel medio, la preparación de trabajos y exposiciones en el marco de eventos o concursos locales que tienen como propósito robustecer la formación científica. La meta principal de articular el abordaje de la nanoquímica bajo el enfoque CTS-A en el bachillerato y nivel superior es formar ciudadanos científicamente cultos, responsables y comprometidos con su entorno.

\section{Conclusiones}

En el contexto educativo, el avance de la nanoquímica ha sido lento debido a la escasa o nula presencia de contenidos relacionados con esta área de conocimientos en los niveles de enseñanza obligatorios. Un problema que está asociado con la complejidad que revisten algunos contenidos presentes en su marco conceptual, así como por la serie de obstáculos epistemológicos que se han reportado en la literatura en los procesos de enseñanza y divulgación del mundo de lo nano (Serena, 2013; Sánchez-Mora y Tagüeña, 2011). Otro ele-

la filosofía y la naturaleza de la ciencia adquirieron relevancia en el ámbito educativo. Entre sus objetivos principales se encuentra la conceptualización de la ciencia como una actividad humana en construcción, desarrollar actividades y prácticas democráticas en torno a la investigación tecnológica, promover la construcción de puentes entre la cultura humanista y el pensamiento científico, vincular los proceso de enseñanza de la ciencia con el desarrollo sostenible, así como fomentar la toma de decisiones informadas en materia de ciencia y tecnología (Vilches et al., 2011; Santos, 2019). 
mento a considerar en torno a esta problemática es que las reformas curriculares suelen ser procesos lentos e independientes de los avances y las transformaciones que experimenta el conocimiento científico. No obstante, el pronunciado avance que ha tenido el campo de la nanoquímica en particular y de las nanociencias en general, invita a considerar como necesaria la inclusión de esta subdisciplina como un eje renovador de los currículos de química, sobre todo en los niveles medio y superior. Esto redituará en una actualización del proceso de enseñanza-aprendizaje de la denominada ciencia central, en la adquisición de una opinión crítica e informada sobre esta área de conocimientos en la ciudadanía y en la formación de vocaciones científicas en el campo.

En el ámbito universitario, el rezago en el tratamiento de aspectos relacionados con la nanoquímica es menor en comparación con lo descrito en la educación obligatoria. Prácticamente, la mayoría de las universidades en el mundo que imparten carreras científicas poseen grados y especializaciones que certifican los estudios en esta área. A pesar de ello, algunos expertos señalan como una dificultad en la enseñanza de la nanoquímica, la falta de homogeneidad en el tratamiento de contenidos, ausencia de estándares tanto de aprendizaje como de evaluación y la desarticulación de conocimientos disciplinares y contextuales (Jones y Blonder, 2013). Para afrontar estos problemas en Europa y EUA se están consolidando cursos intensivos en las etapas iniciales y de pregrado donde se fusionan actividades de lectura, diseño e impartición de conferencias, trabajo de capacitación en el laboratorio, análisis de implicaciones socioambientales, elaboración de artículos científicos y escenarios de debate (Ozin y Cademartiri, 2011; Pagliario, 2015).

Lo presentado en este artículo es una aportación con la intención de robustecer la discusión sobre la relevancia disciplinar de la nanoquímica y la necesidad de promover el trabajo educativo sobre este campo en todos los niveles de estudio. Por último, se puede aseverar que la inclusión de la nanoquímica como un elemento o eje renovador en la educación química en general es una cuestión loable y pertinente. Esto, si se toma en cuenta que actualizar, diversificar y mejorar la calidad de los procesos relacionados con la enseñanza-aprendizaje de la ciencia es una meta inherente de toda institución educativa.

\section{Referencias}

Aegerter, M., Almeida, R., Soutar, A., Tadanaga, K., Yang, H. y Watanabe, T. (2008). Coatings made by sol-gel and chemical nanotechnology. Journal of Sol-Gel Science and Technology, 47(2): 203-236.

Aguayo-González, F., Zarzuela Roldán, E. y Lama-Ruiz, J. (2011). Nanotecnología y nanoquímica. Sevilla Técnica, 36: 28-37. http://hdl.handle.net/11441/38339

Alonso-Núñez, G. (2008). Nanoquímica: ingeniería de nanomateriales. Mundo Nano. Revista Interdisciplinaria en Nanociencias y Nanotecnología, 1(1): 45-50. https:// doi.org/10.22201/ceiich.24485691e.2008.1.53559 
Álvarez-Amparán, M. y Cedeño-Caero, L. (2020). Análisis y relevancia de los procesos catalíticos para la remoción de azufre en los combustibles fósiles. Mundo Nano. Revista Interdisciplinaria en Nanociencias y Nanotecnología, 14(26): 51-71. https://doi.org/10.22201/ceiich.24485691e.2021.26.69633

Atkins, P. (2013). What is chemistry? UK: Oxford University Press.

Bäuerle, D. (2013). Laser processing and chemistry. Springer Science \& Business Media.

Bayda, S., Adeel, M., Tuccinardi, T., Cordani, M. y Rizzolio, F. (2020). The history of nanoscience and nanotechnology: From chemical-physical applications to nanomedicine. Molecules, 25(1): 112. https://doi.org/10.3390/molecules25010112

Bensaude-Vincent, B. (2004). Two cultures of nanotecnology?, HYLE-International Journal for the Philosophy Chemestry, 10(2): 65-82.

https://doi.org/10.1142/9789812773975_0002

Bolaños, L. y Álvarez, X. (2018). Polímeros conductores: aplicaciones en celdas fotovoltaicas y dispositivos electrónicos. Revista de Ciencia y Tecnología, Revista de la Universidad de Costa Rica, 34(1), 18-38.

Bueno, O. (2004). The Drexler-Smalley debate on nanotechnology: incommensurability at work? HYLE-International Journal for the Philosophy Chemestry, 10(2): 65-82. https://doi.org/10.1142/9789812773975_0003

Camacho, B. A., Martínez, O., Arenas, M., Argueta, L., de la Fuente, J. y Acosta, L. S. (2015). Copper: Synthesis techniques in nanoscale and powerful application as an antimicrobial agent. Journal of Nanomaterials, ID 415238, 1-10. https:// doi.org/10.1155/2015/415238

Castro, E., García, A. H., Zavala, G. y Echegoyen, L. (2017). Fullerenes in biology and medicine. Journal of Materials Chemistry B, 5(32), 6523-6535. https://doi. org/10.1039/C7TB00855D

Contreras, R. y Cardozo, E. (2015). Conceptos de nanoquímica. En Laréz-Velásquez, C., Koteich-Katib, S. y López-González, F. (eds.), Nanotecnología: fundamentos y aplicaciones. Mérida, Venezuela: Universidad de los Andes.

Cruz-Alonso, M., Fernández, B., García, M., González-Iglesias, H. y Pereiro, R. (2018). Quantitative imaging of specific proteins in the human retina by laser ablation ICPMS using bioconjugated metal nanoclusters as labels. Analytical chemistry, 90(20): 12145-12151. https://doi.org/10.1021/acs.analchem.8b03124

De Jong, K. P. (2009). Synthesis of solid catalysts. Weinhem: Wiley-VCH.

Delgado, G. C. (2011). Implicaciones éticas, economicosociales y legales de la nanomedicina: el caso de México. En Arnaldi, Simone, Delgado, Gian Carlo, Piccinni, Mariassunta, Poletti, Piera (eds.), Nanomedicina. Entre políticas públicas y necesidades privadas. CEIICH, UNAM/CIGA, Universidad de Padua. México: 67-112.

Delgado, G. C., y Magaña, E. L. (2012). Diálogo para el avance científico y tecnológico a la nanoescala. Mundo Nano. Revista Interdisciplinaria en Nanociencias y Nanotecnología,5(1):82-102.https://doi.org/10.22201/ceiich.24485691e.2012.1.45168

Díaz, G. (2011). Catálisis y nanocatálisis. En Takeuchi, N. (ed.), Nanociencia y nanotecnología. Panorama actual en México. México: CEIICH-UNAM, 43-63. 
Escobar-Alarcón, L. y Solís-Casados, D. (2020). Desarrollo de fotocatalizadores basados en $\mathrm{TiO}_{2}$ en forma de película delgada para la degradación de moléculas orgánicas en solución acuosa. Mundo Nano. Revista Interdisciplinaria en Nanociencias y Nanotecnología, 14(26), 11-33.https://doi.org/10.22201/ceiich.24485691e.2021.26.69646

Feynman, R. P. (1960). There's plenty of room at the bottom. Engineering and Science magazine (Caltech), 23: 22-36.

Fesenko, O. y Yatsenko, L. (2018). Nanochemistry, biotechnology, nanomaterials, and their applications. Springer International Publishing.

Foladori, G. y Invernizzi, N. (2018). A critical vision of disruptive nanotechnologies. Perspectives on Global Development and Technology, 17(5-6): 614-631. https:// doi.org/10.1163/15691497-12341497

García-Betancourt, M. (2017). Perspectivas para la innovación en educación con nanociencia y nanotecnología. Revista de educación y desarrollo, 41: 93-101. Universidad de Guadalajara.

García, A. (2012). Aprendiendo del futuro: gobernando la nanotecnología. CTS: Revista iberoamericana de ciencia, tecnología y sociedad, 7(20), 261-272.

García, N. G. (2013). Electrospinning: una técnica fascinante para la obtención de nanofibras poliméricas. Revista de plásticos modernos: Ciencia y tecnología de polímeros, (677): 166-173.

Isaza, A., Patiño, S., Arango, E. y Rave, N. (2019). Sistema de protección térmica basado en polímeros reforzados con nanotubos de carbono y dióxido de titanio para aplicaciones industriales. Materiales Compuestos, 3(4): 11-16.

Jones, M., Blonder, R., Gardner, G., Albe, V., Falvo, M. y Chevrier, J. (2013). Nanotechnology and nanoscale science: educational challenges. International Journal of Science Education, 35 (9): 1490-1512. https://doi.org/10.1080/0950069 3.2013.771828

Kaplan, S. y Radin, J. (2011). Bounding an emerging technology: Para-scientific media and the Drexler-Smalley debate about nanotechnology. Social Studies of Science, 41(4): 457-485. https://doi.org/10.1177/0306312711402722

Karousis, N., Tagmatarchis, N. y Tasis, D. (2010). Current progress on the chemical modification of carbon nanotubes. Chemical reviews, 110(9): 5366-5397. https:// doi.org/10.1021/cr100018g

López-Quintela, M. (2015). Nanoquímica: una verdadera revolución científico-tecnológica. Panorama social, (21): 39-50.

Martín, N. (2011). Sobre fullerenos, nanotubos de carbono y grafenos. Arbor, 187(Extra_1): 115-131. https://doi.org/10.3989/arbor.2011.extran1117

Martín, J., Camacho, M. y Vega, J. (2013). Influencia de las nanopartículas de sílice en polímeros termoplásticos. Mundo Nano. Revista Interdisciplinaria en Nanociencias y Nanotecnología, 6(11): 40-51. https://doi.org/10.22201/ ceiich.24485691e.2013.11.50003

Meinguer, J. (2018). El valor del pensamiento crítico en la educación científica. Eutopía, 11(29): 5-11. CCH-UNAM.

Meinguer, J. (2019). La comunicación de la nanotecnología a través del análisis crítico de textos informales. Mundo Nano. Revista Interdisciplinaria en Nanociencia y Nanotec- 
nología, 12(22): 3-35. https://doi.org/10.22201/ceiich.24485691e.2019.22.61953

Meinguer, J. y Pérez, M.C. (2021). Desarrollo y evaluación de elementos de pensamiento crítico sobre la química verde en el bachillerato. Investigación en la Escuela, 103: 106-124. https://doi.org/10.12795/IE.2021.i103.08

Munuce, A. (2014). Biografía no autorizada de las nanopartículas. En Silvestri, S., Munuce, A. y Alassia, M., Seferian, A., Reviglio A. y Soria, L., Nanotecnología hoy: el desafío de conocer y enseñar. Buenos Aires: Ministerio de Educación de la Nación, 41-63.

Nevárez-Martínez, M., Espinoza-Montero, P., Quiroz-Chávez, F. y Ohtani, B. (2017). Fotocatálisis: inicio, actualidad y perspectivas a través del $\mathrm{TiO}_{2}$. Avances en Química, 12(2-3): 45-59.

ONU (2019). Informe Mundial sobre el Desarrollo Sostenible 2019: El futuro es ahorala ciencia al servicio del desarrollo sostenible-. Grupo independiente de científicos designados por el secretario general. Naciones Unidas.

Ozin, G. y Cademartiri, L. (2011). From ideas to innovation: nanochemistry as a case study. Small, 7(1): 49-54. https://doi.org/10.1002/smll.201001097

Ozin, G. y Arsenault, A. (2015). Nanochemistry: a chemical approach to nanomaterials. UK: Royal Society of Chemistry Publishing.

Palma, M., Acuña, R., Acosta, G. y Padrón, G. (2010). Estado del arte del proceso solgel en México. CIENCIA ergo-sum, 17(2): 183-188.

Pagliaro, M. (2015). Advancing nanochemistry education. Chemistry-A European Journal, 21(34): 11931-11936. https://doi.org/10.1002/chem.201501042

Pyrrho, M., y Schramm, F. R. (2019). Cruces entre salud colectiva y bioética: la nanotecnología como objeto-modelo. Revista Bioética, 27(4): 587-594. https://doi. org/10.1590/1983-80422019274341

Reviglio, A. 2014. Por qué y para qué enseñar nanotecnología en las escuelas. En Silvestri, S., Munuce, A. y Alassia, M., Seferian, A., Reviglio A. y Soria, L., Nanotecnología hoy: el desafío de conocer y enseñar. Buenos Aires: Ministerio de Educación de la Nación, 159-183.

Ribeiro, A., Godoy, G., Neto, L. y de Souza-Filho, M. (2018). Holografía y realidad virtual en la enseñanza de nanotecnología: nuevos horizontes dirigidos a educación secundaria. MOMENTO, (56E): 34-45. https://revistas.unal.edu.co/index.php/momento/article/view/71645

Ribeiro, A. y de Souza-Filho, M. (2014). Proyecto de ambientes innovadores de enseñanza y la propuesta del laboratorio de formación interdisciplinar de educadores en nanociencia y nanotecnologia-LIFENano/IFSP. MOMENTO, (49E): 38-48. https://repositorio.unal.edu.co/handle/unal/67391

Rojas, M. (2015). Diseño y síntesis de materiales "a medida" mediante el método sol-gel. Madrid: UNED.

Saldívar, L. (2021). Recomendaciones de política pública de nanociencia y nanotecnología en México: privilegiar el bienestar humano y ambiental. Mundo Nano. Revista Interdisciplinaria en Nanociencias y Nanotecnología, 15(28): 1e-23e. https://doi.org/10.22201/ceiich.24485691e.2022.28.69655

Santos, R. y Auler, D. (2019). Práticas educativas CTS: busca de uma participação 
social para além da avaliação de impactos da Ciência-Tecnologia na Sociedade. Ciência \& Educação (Bauru), 25(2): 485-503. https://doi.org/10.1590/1516731320190020013

Sánchez-Mora, C. y Tagüeña, J. 2011. El manejo de las escalas como obstáculo epistemológico en la divulgación de la nanociencia. Mundo Nano. Revista Interdisciplinaria en Nanociencias y Nanotecnología, 4(2): 83-102. https://doi.org/10.22201/ ceiich.24485691e.2011.2.45011

Sebastián, V. (2018). Nanocristales, nuevos materiales con propiedades y aplicaciones únicas. Enseñanza de las Ciencias de la Tierra, 26(3): 306-314. https://www. raco.cat/index.php/ECT/article/view/343169

Serena, P. 2013. Acercando la nanotecnología a la sociedad: la exposición un paseo por el nanomundo. Revista Digital Universitaria, 14 (4). http://www.revista. unam.mx/vol.14/num4/art29/index.html

Serena, P., Giraldo, N., Takeuchi, N. y Tutor J. (2014). Guía didáctica para la enseñanza de la nanotecnología en la educación secundaria. Madrid: nanoDYF. https://cutt. ly/qcCRR3M

Strand, Roger y Nydal, Rune. (2008). Nanoética buena-nanotecnología buena. Mundo Nano. Revista Interdisciplinaria de Nanociencia y Nanotecnología, 1(1): 61-79. https://doi.org/10.22201/ceiich.24485691e.2008.1.53562

Takeuchi, N. (2011). Nanociencia y nanotecnología: la construcción de un mundo mejor átomo por átomo. México: FCE-UNAM.

Vilches, A., Gil, D. y Paia, J. (2011). De CTS a CTSA: Educación por un futuro sostenible. En Pereira., W y Auler, D. (coords.), CTS e educação científica: desafios, tendências e resultados de pesquisa. Brasil: Universidade de Brasília, 185-209.

Zanella, R. (2012). Metodologías para la síntesis de nanopartículas: controlando forma y tamaño. Mundo Nano. Revista Interdisciplinaria en Nanociencias y Nanotecnología, 5(1),69-80. https://doi.org/10.22201/ceiich.24485691e.2012.1.45167 